Juego, derechos y ciudadanía: la ludoteca como ámbito de intervención social

María Victoria Martin, Nancy Díaz Larrañaga y Mónica Zapatería

http://perio.unlp.edu.ar/ojs/index.php/question/article/view/4597

Cita sugerida: Martin, M., Díaz Larrañaga, N., y Zapatería, M. (2018). Juego, derechos y ciudadanía: la ludoteca como

ámbito de intervención social. Question, 1(58), e040. doi:https://doi.org/10.24215/16696581e040

\title{
Juego, derechos y ciudadanía: la ludoteca como ámbito de intervención social
}

Game, rights and citizenship: the toy library as ambit of social intervention

María Victoria Martin mvmartin@perio.unlp.edu.ar

http://orcid.org/0000-0001-6249-6935

Instituto de Investigaciones Sociosimbólicas Latinoamericanas Aníbal Ford; Facultad de Periodismo y Comunicación Social; Universidad Nacional de La Plata/ Universidad Nacional de Quilmes (Argentina)

Nancy Díaz Larrañaga nlarran@perio.unlp.edu.ar http://orcid.org/0000-0001-9727-4718

Instituto de Investigaciones Sociosimbólicas Latinoamericanas Aníbal Ford; Facultad de Periodismo y Comunicación Social; Universidad Nacional de La Plata/ Universidad Nacional de Quilmes (Argentina)

Mónica Zapatería monicazapateria@gmail.com http://orcid.org/0000-0003-2759-1333

Facultad de Periodismo y Comunicación Social;

Universidad Nacional de La Plata (Argentina) 


\section{Resumen}

En este artículo se reflexiona sobre la importancia del juego como ámbito de desarrollo de los derechos del niño y como práctica social, a partir del análisis de la experiencia de la ludoteca La Máquina de los Sueños, del barrio San Carlos de la ciudad de La Plata. A su vez, y en relación con la ciudadanía, se entiende a ese ámbito como práctica de intervención social que propicia el cambio social.

El trabajo se enmarca en el proyecto de investigación "Prácticas sociales y representaciones temporales: cruces entre lo educativo/político y el cambio social" (2015-2019), Facultad de Periodismo y Comunicación Social, Universidad Nacional de La Plata, Argentina.

Palabras clave: juego; ludoteca; derechos; intervención; cambio social.

\section{Abstract}

The purpose of this article is to reflect on the importance of game as ambit of Rights of the Child development and social practice. It analyse the experience of La Máquina de los Sueños, a toy library settled in San Carlos neigbourhood, in La Plata. In adittion, related to citizenship, we understand that this ambit proposes social intervention in orden to social change.

The study is part of the research project "Social practices and temporary representations: interchange between educational/political issues and social change" (2015-2019), Facultad de Periodismo y Comunicación Social, Universidad Nacional de La Plata, Argentina.

Keywords: game; toy library; rights; intervention; social change.

La primera ludoteca se crea en 1934 en Los Ángeles, con una función compensatoria similar a la de las bibliotecas públicas: prestar juegos y juguetes a niños en situación de vulnerabilidad para que pudieran utilizar en sus casas. A partir de la década del sesenta, la Organización de las Naciones Unidad para la Educación, la Ciencia y la Cultura (UNESCO) promueve estos espacios facilitadores del juego y de juguetes, motivo por el cual se expanden por casi todos los países.

En Latinoamérica los antecedentes se remontan a la década del setenta con proyectos en Brasil, Uruguay, Perú, Argentina, Cuba y Costa Rica. En estos últimos cinco años se suman experiencias en Colombia, Ecuador, Paraguay, Bolivia, México, Panamá, Venezuela y Honduras. En la actualidad, es importante el movimiento ludotecario en Francia, España, Gran 
Bretaña, Australia, Italia y Portugal, en muchos casos promovido y apoyado por el mismo Estado.

La Máquina de los Sueños (1) es una asociación civil sin fines de lucro fundada en 1998 en la ciudad de La Plata. Sus objetivos principales son brindar apoyo moral, educativo y material a la niñez en situación de abandono, aislamiento, desfavorabilidad social o económica, o discapacidad y propiciar también el acercamiento de esos niños y jóvenes al arte y la técnica para generar competencias que permitan una futura inserción en el mundo del trabajo y generar proyectos para adultos tendientes a la formación profesional y laboral.

Actualmente, tiene en funcionamiento un jardín de infantes, una ludoteca, una bebeteca, un programa de padrinazgos escolares, una biblioteca y talleres de apoyo escolar. Funciona en la calle 148 entre 35 y 36 (Barrio San Carlos de la ciudad de La Plata). La bebeteca comienza a funcionar unos años después, en 2011, gracias a un convenio con el Instituto Superior de Formación Docente Nro. 17 de La Plata. Graciela Cavalieri, fundadora del lugar y por entonces docente de esa institución educativa, impulsa la formación como ludotecarios en territorio para los estudiantes de primer año del Profesorado en Educación Inicial.

La asociación atiende principalmente a los vecinos de la zona, en su mayoría migrantes internos o de países limítrofes, además de miembros de una comunidad toba próxima a la sede. Se trata de una población caracterizada por la precarización e informalidad laboral, con problemáticas sociales que se derivan de esa situación y afecciones a la salud derivadas de factores ambientales y de infraestructura (en especial, por la carencia de agua potable).

Numerosas investigaciones afirman que los primeros años de vida resultan decisivos para el desarrollo posterior de la persona, ya que constituyen un período crítico de sus necesidades biológicas, psicológicas y sociales en relación central con la alimentación, la salud, el desarrollo de su inteligencia, personalidad y afectos. Algunos estudios resaltan la vulnerabilidad de esos grupos de edad en condiciones desfavorables del "ambiente físico y social, tales como la falta de estimulación psicosocial, intelectual y la falta de cariño; por tanto, la educación extraescolar debe ser una prioridad de cualquier programa que busque impulsar el desarrollo digno e integral de la infancia" (Ulloa Ziaurriz, 2000: 9).

\section{Locales para jugar}

El concepto ludoteca proviene del latín y conjuga "ludos" que significa juego y "theca", caja o local para guardar algo. Bajo múltiples expresiones, los especialistas coinciden en señalar que 
una ludoteca constituye un ámbito de encuentro entre niños; que tiene diversos juguetes destinados al juego de los chicos o a su préstamo; que ha de ser abierta a distintos sectores sociales y que cuenta con un agente de intervención (el ludotecario), que se desempeña como el educador del lugar (Monroy Antón y Sáez Rodríguez, 2011: 2). Por su parte, Bianchi Zizzias las define como "unidades didácticas-recreativas-culturales que tienen como objetivo el desarrollo de la personalidad del niño a través del juego y el juguete" (1995: 15).

Con una función más amplia que en el comienzo, además de la "diversión" las ludotecas contemporáneas buscan facilitar las relaciones sociales, estimular el desarrollo integral de los niños, en especial en contextos desfavorables o de vulnerabilidad, como el del barrio San Carlos.

Existen distintos tipos de ludotecas: públicas, privadas o de asociaciones y ONG; especializadas en niños con discapacidad, terapéuticas, de investigación, hospitalarias, escolares, en cárceles, móviles, eventuales; bebetecas (con zonas de movimiento, psicomotricidad, juego simbólico y de imitación, construcciones y rompecabezas, expresión plástica; con participación de madres/padres); ludotecas infantiles (destinadas a la recreación deportiva, juegos de movimiento, juegos simbólicos y dramatizaciones, construcciones, juegos de mesa y de sociedad, taller, biblioteca recreativa, paseos y reconocimiento del entorno y la ciudad) y ludotecas juveniles (con menos experiencias, podemos relacionarlas -además de las propuestas anteriores- con juegos vinculados a la prevención y promoción de la salud; la independencia y autonomía, los derechos, la seguridad y privacidad online, y otras áreas de interés y conflictividad para ese grupo).

En el caso singular de nuestro estudio, se trata de una práctica de educación no formal, destinada a niños de entre tres y doce años. En este espacio lúdico se entiende que el juego es liberador; que enseña, unifica, tiene funciones sociales y lingüísticas fundamentales. Durante 2017, Graciela Cavalieri (2), fundadora y principal referente de la organización, promovió dos reuniones de perfeccionamiento sólo con las alumnas del primer año del Instituto de Formación Docente Nro. 17 de La Plata que realizan su práctica como ludotecarias en La Máquina de los Sueños. En las mismas destacó el trabajo cognitivo que implican los juegos, trabajo semejante al aprendizaje escolar, pero que se diferencia porque en la ludoteca priorizan que el chico se divierta y aprenda. Se proponen, entonces, trabajar las cuestiones cognitivas a través del juego, dejando en un segundo plano las estrategias de aprendizaje escolar.

Sobre la incidencia de las ludotecas en el desarrollo integral de las niñas y los niños, los lineamientos del gobierno de México, que instituye programas especiales, expresan: 
En un espacio lúdico se forman y desarrollan sus capacidades individuales a través de actividades diseñadas pensando en las habilidades y capacidades físicas, psicológicas, sensoriales y cognoscitivas. Asimismo, la ludoteca es formativa en el aspecto social de la niñez, pues jugando en grupo aprenden a respetar las reglas necesarias para la convivencia, a ayudar y recibir ayuda, a cooperar y a comprender a las otras y los otros. Además, pueden convertirse en un medio para atenuar diferencias socioculturales. A partir del juego y el juguete, los niños y niñas pueden aprender el respeto, habituándose a considerarlo como un valor indispensable y necesario para una mejor convivencia. Las ludotecas despiertan un sentimiento de responsabilidad y de vida social; desarrollan una nueva relación gracias al objeto que no se posee, sino que se comparte (Ulloa Ziaurriz, 2000: 7).

Graciela Cavalieri explica que los chicos realizan juegos de mesa (cartas, tableros, bingos, entre otros) a través de los cuales desarrollan distintas habilidades, incluso suman y restan sin darse cuenta, aprenden formas de registros. En otro orden, aparecen los juegos motrices, de carreras, de educación física, inventan juegos en el espacio con aros; en el verano, desarrollan juegos al aire libre y con agua, todos con mucha aceptación.

En la ludoteca hay también una cocina para niños en donde aprenden, desde la curiosidad y el entretenimiento, las medidas, qué le pasa a determinada sustancia, si se disuelve o no, y observan los procesos químicos. En cuanto a la labor realizada en la ludoteca de bebés, a la que asisten niños de seis meses a tres años, se destaca que cubren a principios de año, en dos horas, el cupo disponible de veinte bebés y luego organizan una larga lista de espera. Además, atienden derivados por los centros de salud casos de niños que padecen de alimentación pobre, falta de estimulación temprana e hipotónicos, a los que se les da prioridad ante la ausencia en el barrio de un sitio que contenga estas situaciones más complejas. De la bebeteca participan mayormente madres, a quienes se las estimula para jugar con sus niños.

\section{El juego como derecho}

El juego infantil se define como una actividad placentera, libre y espontánea, sin un fin determinado, pero de gran utilidad para el desarrollo del niño: "A través del juego los y las preescolares pueden representar la identidad o las características de él o ella, de otra persona, objeto, de una acción o situación" (Craig y Baucum; 2001: 227).

Igualmente, el juego tiene una función pedagógica: mediante el mismo, las niñas y los niños pueden aprender y de esta manera contribuir al fortalecimiento de su personalidad en un 
ambiente que ofrezca espacios para que cada uno encuentre su lugar, tome la iniciativa, descubra sus propias capacidades, respete al otro, valore los esfuerzos propios y ajenos (Sáenz López, 2014: 34). El juego favorece el "saber y hacer" que tiene el niño para desenvolverse en distintos ambientes y para resolver nuevos problemas, por lo que se to ha considerado como una actividad irremplazable en el aprendizaje del niño.

Para Bruner, el juego constituye una actitud con respecto al uso de la inteligencia mediante la cual se experimentan formas de combinar el pensamiento, el lenguaje y la fantasía. Además, "el juego libre ofrece al niño la oportunidad inicial y más importante de atreverse a pensar, a hablar y quizás incluso de ser él mismo" (1986: 85).

A partir de la Declaración de los Derechos del Niño, promulgado unánimemente por la Asamblea General de las Naciones Unidas en 1959, el juego constituye uno de los derechos del Niño: "El niño debe disfrutar plenamente de juegos y recreaciones, los cuales deberán estar orientados hacia los fines perseguidos por la educación; la sociedad y las autoridades públicas se esforzarán por promover el goce de este derecho".

Aunque el juego constituye una capacidad innata, su desarrollo depende del estímulo del entorno y en un primer momento de vida, interviene principalmente la familia. En contextos de vulnerabilidad y carencia, es importante poder apuntalar a los miembros adultos para hacerlo, ya que es a través del juego que los niños desarrollan su personalidad y, con esto, su lugar en el mundo. Por eso son los adultos quienes deben garantizar este derecho, propiciando los ámbitos, espacios y momentos para el juego. En la ludoteca de La Máquina de los Sueños, en especial en la bebeteca, se enseña a las madres a jugar con sus hijos. Involucrar a las familias permite construir vínculos singulares, resignificando la relación madre/niño, en especial porque muchas madres no han tenido la oportunidad de jugar con sus propios padres por situaciones de abandono, falta de tiempo, entre otras.

Por su parte, la International Play Association (IPA), organización reconocida por el Consejo Económico y Social de las Naciones Unidas (ECOSOC), con estatus consultivo con UNESCO y UNICEF, cuyo principal objetivo es preservar, proteger y promover el derecho de todos los niños y niñas al juego, destaca "la creciente evidencia de los efectos de la falta de tiempo y espacio para el juego sobre los cuerpos y las mentes de los niños", por lo que aprueba en su reunión de Barcelona de 1989 la siguiente declaración (3):

-Los Niños son la base del futuro del mundo.

-Los Niños han jugado a través de todos los tiempos y en todas las culturas.

-El Juego, junto con los requerimientos básicos de nutrición, salud, vivienda y educación constituye un área vital del desarrollo del potencial de todos los niños. 
-El Juego es comunicación y expresión, combinando pensamiento y acción, da satisfacción y sensación de logro.

-El Juego es instintivo, voluntario y espontáneo.

-El Juego ayuda al desarrollo físico, mental, social y emocional del niño.

-El Juego es una forma de aprender a vivir y no un mero pasatiempo.

En sintonía con ambas declaraciones, la Convención de los Derechos del Niño, adoptada por la Asamblea General de las Naciones Unidas en 1989, establece que el niño tiene derecho al tiempo libre, al juego y a participar en actividades culturales y artísticas.

\section{El juego como práctica sociocultural}

Más allá de estos manifiestos, el juego es una práctica cultural y social plenamente asociada a la educación, sobre todo en los primeros años de vida de un niño. La noción de "aprender jugando" es central en el nivel inicial del sistema educativo, en el que se propone la apropiación de saberes desde lo lúdico. Lamentablemente, nuestra sociedad prioriza el saber racional sobre otros tipos de saberes y, paulatinamente con el crecimiento, el juego se va abandonando y desvaneciendo en los otros niveles educativos para dar lugar al trabajo abstracto a partir de la conceptualización.

El juego es una práctica libre que se encuentra delimitada en un espacio y un tiempo. A su vez, está reglamentada en tanto que el propio juego presenta reglas (como podría ser el reglamento que acompaña un juego de mesa) o las establecidas por los jugadores que las construyen a partir de acuerdos y consensos. Estas dos dimensiones, el encuadre espaciotemporal y las reglas, permiten que esta práctica "libre" pueda utilizarse como una estrategia didáctica para otros fines, además del de jugar.

Al ubicarse el juego entre lo real y lo simbólico, el niño puede recorrer desde múltiples aproximaciones un mismo fenómeno o tema; potencia la creatividad y pone en común, si el juego es colectivo, sentidos en un acto comunicativo pleno.

Por medio del involucramiento en el juego se vivencian emociones y tensiones que reconfiguran los modos de jugar y a veces sus reglas. Estas reglas son los elementos básicos de la norma social, posibilitan que los chicos jueguen juntos, compartan y participen de procesos comunicativos entre ellos y con los adultos que también juegan. Desde este lugar 
podemos pensar la importancia del juego en tanto saber jugar, pero también el juego como saber.

La relevancia pedagógica del juego radica en brindar la posibilidad de ocupar distintos roles: ser partícipe del juego o gestor del mismo, motivando así el rol activo frente al conocimiento, a la construcción de las reglas y a la construcción del sentido del mundo desde lo simbólico, pero también desde lo real.

Por este motivo podemos pensar al juego desde una dimensión superficial, la lúdica pero también desde otra dimensión profunda, la construcción de subjetividad. Esta distinción entre superficial y profundo no responde a valoraciones, sino a lo que aparece a primera vista y a lo que se encuentra un tanto más oculto.

Los espacios institucionalizados, como las ludotecas, presentan varios desafíos que necesitan resolver en el día a día. El primero de ellos es debatir el rol del juego en las vidas de los sujetos y sus comunidades, su "utilidad/inutilidad", para volver a jerarquizar este espacio que valoramos en los niños pero que nos cuesta valorar en la adultez tal vez por la influencia de los modos de aprender que hemos internalizado. El segundo desafío es poner en diálogo la doble normativa: la institucional (de la ludoteca) y la propia del juego, que deben convivir sin cercenar la creatividad y la apropiación de los participantes.

Por último, la tensión entre un juego ritualizado y la apropiación transformadora, que permita la proactividad y el desafío de pensar un mundo distinto.

\section{El juego y la ciudadanía}

Podemos afirmar que jugar construye ciudadanía. Para ello es necesario definir qué entendemos por ser ciudadano hoy. Las miradas tradicionales asocian la ciudadanía a ser iguales frente a la ley, lo que implica derechos (sociales y civiles, al voto, a ser representado por los gobernantes, entre otros) y obligaciones. Los derechos deben ser garantizados frente a toda distinción de edad, género, situación socioeconómica, etcétera. Sin embargo, esta noción tradicional homogeniza a los sujetos en tanto los iguala, pero no reconoce sus diferencias constitutivas de las relaciones de poder.

En este sentido, la ciudadanía se presenta como el reconocimiento de nosotros y los otros como sujetos sociales, donde cobra un lugar central la lucha por ese reconocimiento. En este proceso influyen las apropiaciones de bienes materiales y simbólicos que nos ayudan a transitar en nuestras sociedades. Es así que 
ser ciudadano no tiene que ver sólo con los derechos reconocidos por los aparatos estatales a quienes nacieron en un territorio, sino también con las prácticas sociales y culturales que dan sentido de pertenencia y hacen sentir diferentes a quienes poseen una misma lengua, semejantes formas de organizarse y satisfacer sus necesidades (García Canclini, 1995: 19).

Jugar con otros permite el reconocimiento de los jugadores en su doble faceta: como partícipes del juego y como sujetos sociales que comparten momentáneamente un espacio de construcción colectiva. Permitir que todos los jugadores gestionen el juego en algún momento posibilita que internalicemos derechos y deberes, roles, reconocimiento de normas, temporalidades y espacialidades, otros modos de imaginar, otros modos de jugar.

\section{La ludoteca como práctica de intervención}

Desde distintos enfoques, el juego supone una intervención, básicamente en el estímulo y desarrollo de ciertas competencias. El enfoque clásico entiende al juego como "un elemento básico en el desarrollo cognoscitivo del niño: en la construcción del espacio, del tiempo y de la imagen propia"; el enfoque psicológico indica que "durante la niñez el juego cumple la función de interpretar y reorganizar los elementos que existen en el mundo real"; y el enfoque social sostiene que

\footnotetext{
la actividad lúdica es un proceso de educación inserto en la propia vida, completo e indispensable, que comporta al niño facilidad, libertad, entusiasmo y gratificaciones satisfactorias en la transición del pensamiento concreto al abstracto, del instinto a la autonomía creadora y a la moralidad (Bonilla Ramírez y Aguilar Alatorre; 2013: 495).
}

En las conceptualizaciones más difundidas, la intervención se orienta "hacia una noción de acción práctica, enfatizando la dimensión espacial (el terreno, el lugar, el campo) donde se ejerce el quehacer profesional, intentando dar un buen servicio a la gente" (Matus, 2004: 34). En relación directa con las actividades que proponen las ludotecas, en tanto espacios que persiguen el desarrollo de la personalidad del niño a través del juego y el juguete, Bianchi Zizzias resalta que: 
una mirada prospectiva sobre el niño y su derecho a jugar nos sitúa en una reflexión sobre posibilidades, deseos, metas, aspiraciones, necesidades y esperanzas, que debe partir del análisis de la realidad actual del contexto sociocultural del que emerge el niño del futuro (1995: 73).

En este sentido, Cavalieri explica:

Desarrollamos actividades básicas para la vida como son la iniciativa y la creatividad. La iniciativa porque proponemos juegos, variantes de juegos y formas de concluirlos; y la creatividad porque es para la vida y se desarrolla, si te lo proponés, en el juego. Hay que saber cómo hacerlo, lo que se hace en la vida con placer, se aprende y favorece el desarrollo en todo sentido. Nosotros hemos observado chicos que no se incluían en los juegos y ahora ellos los están proponiendo. O sea el proponer juegos, variantes de juegos, proponer formas de subdividirse, de llamar a los equipos, ponerle nombre a tal cosa, eso hace que el chico desarrolle su parte creativa en iniciativa (G. Cavalieri, comunicación personal, noviembre de 2017).

\section{Dos décadas cambiando el mundo}

El cambio social se relaciona con la transformación que surge de la voluntad, que fomenta la participación, el diálogo entre diferentes actores y contextos, estimulando procesos creativos, de toma de decisiones; cimentando confianza, revalorizando el saber local, apoyando el crecimiento e impulsando políticas que emanen de los propios deseos, necesidades e intereses de una comunidad.

Desde una aproximación inicial, es posible conceptualizar el cambio social como las transformaciones de las condiciones de vida de los grupos humanos, de su estructura y de su sistema de valores; siempre en relación con procesos de transformación y/o modificación y/o emancipación (aún con la carga política que trae a colación por su parentesco con nociones como desarrollo).

Para Gumucio Dagron, se debe entender que

la comunicación para el cambio social trabaja esencialmente con culturas y que se necesita una sensibilidad muy especial para apoyar el proceso de cambio social en el mundo en desarrollo, que no tiene nada más a que aferrarse que a su identidad cultural. Desarrollo y 
cambio social deben ser posibles dentro de un proceso de intercambios culturales horizontales y respetuosos (2001: 4).

Si la comunicación resulta central en el cambio social, es en las prácticas en donde se ponen en juego valores, por lo que tienen un grado de conflictividad propia de los procesos en los que existe la interacción y que demandan decisiones éticas y políticas.

Al articular nuestro marco teórico con la práctica seleccionada se ve claramente reflejada la sensibilidad y el proceso de cambio social propuesto por los autores. Son acciones concretas realizadas por la Asociación Civil sin fines de lucro La Máquina de los Sueños desde 1997 a la fecha. Se cumplen veinte años de iniciativas y proyectos concretados, a través de los diversos espacios en los que reciben tanto a bebés como a niños de hasta doce años. Guiadas por principios lúdicos y por su experiencia docente, el grupo de mujeres que conforma la comisión directiva de la organización hablan por medio de sus acciones.

A lo largo de estas décadas, incentivan y estimulan a los chicos a través de actividades recreativas, artísticas y literarias en pos de su desarrollo. Sus tareas trascienden el espacio físico de La Máquina de los Sueños, porque los niños realizan distintos paseos: la Casa de Gobierno, el Teatro Argentino de La Plata, los museos de Ciencias Naturales y Dardo Rocha, la reserva natural de Punta Lara, la casa ecológica del Bosque, el camping del Sindicato de Judiciales, sólo por mencionar algunos sitios.

No están solas en esta ardua tarea de, a través del juego, intentar transformar las realidades y futuros de los niños y de la comunidad próxima; las apoyan y acompañan en la construcción de diversos saberes distintas instituciones y estudiantes y docentes universitarios de distintas facultades de la UNLP (4). En los actos de fin de año o en las cenas que organizan anualmente para recaudar fondos, también cuentan con la ayuda de otras organizaciones civiles con fines solidarios, que se ocupan de la animación y el sonido de las fiestas, como así también de acercar donaciones periódicamente a la ludoteca.

Este año preparan los festejos del vigésimo aniversario y, entre otros proyectos -como la edificación de un aula destinada a la enseñanza de "artes y oficios"- editarán una revista en la que reflejen los sueños y realidades de dos décadas.

\section{Notas}


(1) El nombre se corresponde con el título de un libro publicado en Italia (La Macchina dei Sogni), escrito por el hermano de la fundadora del proyecto, Graciela Cavalieri. Desde entonces, trabajan con Associazione Culturale La Macchina dei Sogni, una organización gemela.

(2) Entrevista realizada en el marco de este proyecto de investigación en noviembre de 2017.

(3) Web de la IPA. http://ipaworld.org/childs-right-to-play/the-childs-right-to-play/

(4) Entre otros, desde la cátedra de Parasitosis de Medicina controlan a los niños a través de análisis clínicos para detectar si están infectados y hacer la derivación correspondiente. Además, realizan dramatizaciones para que los chicos adquieran el hábito de lavarse las manos. Por su parte, estudiantes de Veterinaria brindan charlas sobre el cuidado de las mascotas y la adopción responsable, además de vacunar y castrar a animales de la zona. Tienen, además, convenio con la cátedra de Lengua Inglesa II de la Facultad de Humanidades, a partir de un proyecto multilingüístico para introducir a niños mayores de ocho años en el aprendizaje de algunas palabras básicas en inglés; también con tareas de apoyo escolar en ese área.

\section{Bibliografía}

Antón, A. M. y Rodríguez, G. S. (2011). Concepto y tipos de ludotecas. Lecturas: Educación física y deportes, 161(5).

Assinato, G.; Vestfrid, P. y Teodosio, M. A. (2017). Juego, comunicación y cambio social: una experiencia de ludoteca infantil en La Plata. Question, 1(53), FPyCS, UNLP.

Benito Gadea, V. (2016). Las ludotecas en la educación no formal. Profundizando en un caso. Recuperado de http://uvadoc.uva.es/bitstream/10324/18705/1/TFG-O\%20792.pdf

Bianchi Zizzias, E. (1995). Ludotecas: espacios para jugar de la A a la Z. Buenos Aires: Fundación Educa.

Bonilla Ramírez, A. M. y Aguilar Alatorre, G. (2013). La ludoteca: una experiencia educativa y recreativa no formal para crear oportunidades de aprendizaje, como forma de intervención en niños, jóvenes y adultos. Ponencia presentada en el Primer Congreso Internacional de Intervención Educativa, Universidad Pedagógica Nacional, Sevilla. Recuperado de http://www.academia.edu/download/32408767/libro_lie.pdf\#page=493

Bruner, J. (1986). Juego, pensamiento y lenguaje. Perspectivas, 16(1), 79-85.

Craig, G. J. y Baucum, D. (2001). Desarrollo psicológico. México: Pearson Educación.

Díaz Larrañaga, N.; Martin, M. V. y Echeverría, M. P. (2011). Notas sobre la voluntad de cambio en el espacio público: la importancia de la intervención en comunicación. Anuario de investigaciones 2011. Facultad de Periodismo y Comunicación Social, Universidad Nacional de La Plata, La Plata. Recuperado de http://sedici.unlp.edu.ar/bitstream/handle/10915/62995/Documento_completo_.pdfPDFA.pdf?sequence $=1$ 
García Canclini, N. (1995). Consumidores y Ciudadanos. México: Grijalbo.

Gumucio Dagron, A. (2001). Comunicación para el cambio social: el nuevo comunicador. Recuperado de http://comminit.com/la/node/150367

Matus, T. (2004). Apuntes sobre intervención social. Chile. Recuperado de http://trabajosocialucen.files.wordpress.com/

Sáenz López, N. (2014) Ludoteca Infantil Jugando Aprendo (Trabajo fin de grado). Recuperado de http://biblio3.url.edu.gt/Tesario/2014/05/84/Saenz-Nancy.pdf

Ulloa Ziaurriz, T. (coord.) (2000). El libro de la ludoteca. Una guía para aprender jugando. Dirección general de equidad y desarrollo social del Gobierno del distrito Federal, México.

http://www.sideso.cdmx.gob.mx/documentos/libro_ludoteca_df.pdf 